\title{
MiR-155-5p positively regulates CCL17-induced colon cancer cell migration by targeting RhoA
}

\author{
Amr A. Al-Haidari ${ }^{1}$, Ingvar Syk ${ }^{1}$, Henrik Thorlacius ${ }^{1}$ \\ ${ }^{1}$ Department of Clinical Sciences, Section of Surgery, Lund University, 20502 Malmö, Sweden
}

Correspondence to: Henrik Thorlacius, email: henrik.thorlacius@med.lu.se

Keywords: chemokines, chemotaxis, metastasis, microRNA, colon cancer

Received: November 01, $2016 \quad$ Accepted: January 16, $2017 \quad$ Published: January 27, 2017

\section{ABSTRACT}

Colorectal cancer is the second most common cause of cancer-related death, which is due to migration of tumor cells to distant sites of metastasis. Accumulating data indicate that mciroRNAs play an important role in several aspects of colon cancer cell biology. Herein, we examined the role of miR-155-5p in colon cancer cell migration induced by the CCL17-CCR4 axis in HT-29 colon cancer cells. We found that miR-155-5p knockdown in serum starved colon cancer cells decreased CCL17-induced cell chemotaxis. Moreover, knocking down miR-155-5p markedly decreased CCL17-provoked activation of RhoA in colon cancer cells. Bioinformatics analysis predicted two putative binding sites in the AU-rich element at the $3^{\prime}$-UTR of RhoA mRNA. MiR-155-5p binding to RhoA mRNA was verified using a target site blocker and functionally validated by RNA immunoprecipitation assays, showing that miR-155-5p-dependent regulation of RhoA mRNA is mediated by AU-rich elements present in the 3'-UTR region. Taken together, these results show that miR-155-5p positively regulates RhoA mRNA levels and translation as well as cell migration in serum starved colon cancer cells and indicate that targeting miR-155-5p might be a useful strategy to antagonize colon cancer metastasis.

\section{INTRODUCTION}

Colorectal cancer is the third most prevalent cancer and the second most frequent cause of cancer-related death in the world $[1,2]$. Metastasis is the dominant cause of death in patients with colon cancer [3]. The mechanisms behind colon cancer metastasis remains elusive but accumulating data indicate that increased expression of adhesion molecules and chemokines facilitate colon cancer migration and spread [4]. On top of cell migration, chemokines have been implicated in additional aspects of malignant transformation, such as proliferation, survival, and angiogenesis [5]. Convincing data have shown that colon cancer cells express several types of chemokine receptors. For example, it has recently been reported that colon cancer cells express CCR4, the receptor of CCL17, and that CCR4-CCL17 interactions mediate colon cancer cell migration [6]. Additional studies have demonstrated that the CCR4-CCL17 axis can promote breast cancer metastasis to the lung [7]. Cell migration is a dynamic and complex process involving multiple specific transcription factors, including small $(\sim 21 \mathrm{kDa})$ guanosine triphosphatases of the Ras-homologus (Rho) family, such as Rho A-C, Cdc42, and Rac1[8]. Indeed, CCL17/ CCR4-dependent colon cancer cell migration is associated with increased activity of RhoA and can be inhibited by targeting Rho-kinase function [6]. However, the detailed intracellular mechanisms regulating RhoA-dependent gene translation and chemotaxis are not known.

MicroRNAs (miRNAs) are short non-coding RNAs that function as post-transcriptional regulators of gene expression in effector complexes containing a core argonaute protein (AGO) [9, 10]. In general, miRNAs are predominately considered to cause downregulation of mRNA expression; however, under certain conditions miRNAs can upregulate expression of distinct mRNAs [11-13]. For example, serum starvation, which puts cells in a state of quiescence, has been shown to favor mRNA expression by certain miRNAs [13-15]. MiR-155 is overexpressed in different types of tumors, including colon cancer [16] and high expression of miR-155 correlates with poor prognosis in colorectal cancer patients [17] suggesting a pro-carcinogeneic role of miR-155. Increased RNA translation induced by miRNAs has been shown 
to be mediated via AU-rich elements (AREs) in the 3' untranslated region (UTR) of mRNA for instance AUUA and AUUUA, as well as adjoining non-AU sequences [18] Notably, investigations have reported that miR-155 can regulate migration and invasion of certain tumor cell types, such as breast, pancreatic, hepatocellular and nasopharyngeal cancers [19-22]. However, the role and mechanism of MiR-155 in regulating chemokine-induced colon cancer cell migration is not known.

Based on the considerations above, we hypothesized that should be added after hypothesized miR-155-5p might regulate CCL17-induced and CCR4-dependent migration of colon cancer cells via RhoA function. For this purpose, we used a human colon cancer cell line transfected with AntagomiR-155-5p.

\section{RESULTS}

\section{Downregulation of miR-155-5p inhibits migration of colon cancer cells}

To analyze the function of miR-155-5p in colon cancer cells, miR-155-5p was knocked down in serum starved HT-29 colon adenocarcinoma cell line using AntagomiR-155-5p. Transfection with AntagomiR-155-5p dose-dependently decreased miR-155-5p expression in HT-29 cells (Supplementary Figure 1A). For example, transfection with $200 \mathrm{nM}$ of AntagomiR-155-5p reduced miR-155-5p expression by $77 \%$ (Supplementary Figure 1A). Importantly, AntagomiR-155-5p transfection had no effect on tumor cell viability (Supplementary Figure 1B). To investigate whether suppression of miR-155-5p affects colon cancer cell migration, transwell migration assays were performed with CCL17 as a chemoattractant. We found that transfection with AntagomiR-155-5p abolished CCL17induced colon cancer cell migration compared with control (ctrl) or AntagomiR ctrl (Figure 1).

\section{miR-155-5p inhibition decreases RhoA activity}

Knowing that CCL17-induced colon cancer cell migration is dependent on RhoA signaling [6], we next asked whether miR-155-5p might regulate CCL17-evoked activation of RhoA. It was found that AntagomiR-155-5p transfection markedly decreased RhoA mRNA expression in HT-29 starved cells (Figure 2A) while knocking down miR-155-5p in normal serum conditions increased RhoA mRNA (Figure 2B and Supplementary Figure 1C). Co-incubation with CCL17 significantly increased RhoA activity in HT-29 cells (Figure 2C). Moreover, we observed that knocking down miR-155-5p also abolished CCL17-induced activation of RhoA (Figure 2C).

\section{Target prediction of miR-155-5p}

We asked if RhoA mRNA could be a direct target for miR-155-5p in HT-29 cells and whether the positive regulation profile on cell migration is related to direct binding of miR-155-5p to RhoA mRNA. RNAhybrid target prediction analysis revealed five different target hits in the $3^{\prime}$-UTR of RhoA mRNA containing

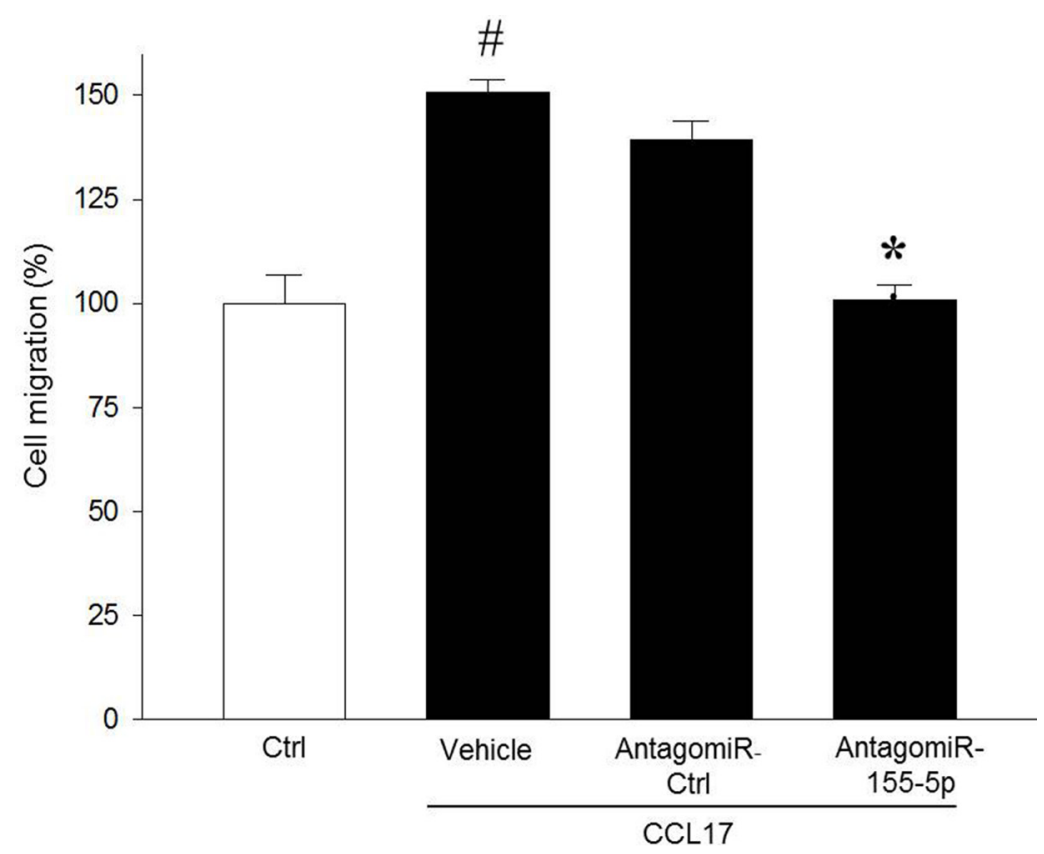

Figure 1: MiR-155-5p regulates colon cancer cell migration. AntagomiR-155-5p (200 nM) decreased CCL17-induced HT-29 colon cancer cell migration. Cells were counted microscopically using 10 High Power Fields in five different fields. Migration index was calculated as the ratio of the number of migrated cells on wells containing CCL17 divided by the number of cells in the control wells. ${ }^{\#} P<0.05$ versus negative ctrl and $* P<0.05$ versus CCL17+ AntagomiR ctrl. All data are expressed as mean $\pm \mathrm{SEM}$ and $n=4$. 
miR-155-5p recognition sites (Supplementary Figure 2). However, based on potentially existing evidences on the role of ARE sites in RNA translation, [18] we limited our analysis to the regulatory ARE sites in the 3'-UTR of RhoA mRNA. Strikingly, we found two potential sites, i.e., target site 1 containing the tetramer AUUA which is complementary to the $4^{\prime}$-mer of the miR-155-5p seeding region and target site 2 containing the tetramer AUUA and the pentamer AUUUA (Figure 3A and 3B). The function of these sites was examined by use of TSBs. Again, it was found that transfection with AntagomiR155-5p markedly reduced RhoA mRNA expression in HT-29 (Figure 3C). Interestingly, we observed that co-transfection with TSB1 which blocks target site 1 was unable to reverse the effect of AntagomiR-155-5p (Figure 3C). In contrast, co-transfection with TSB2, which blocks target site 2, dose-dependently increased expression of RhoA mRNA in HT-29 carcinoma cells transfected with AntagomiR-155-5p (Figure 3C), suggesting that miR-155-5p interacts with this specific target site in RhoA mRNA.

\section{RhoA mRNA is a direct target of miR-155-5p}

To verify the association between miR-155-5p and RhoA mRNA in HT-29 cells, we used RIP with antiAgo- 2 beads. The Ago 2 protein is the core and catalytic component of RISC [24]. Since microRNAs are known to
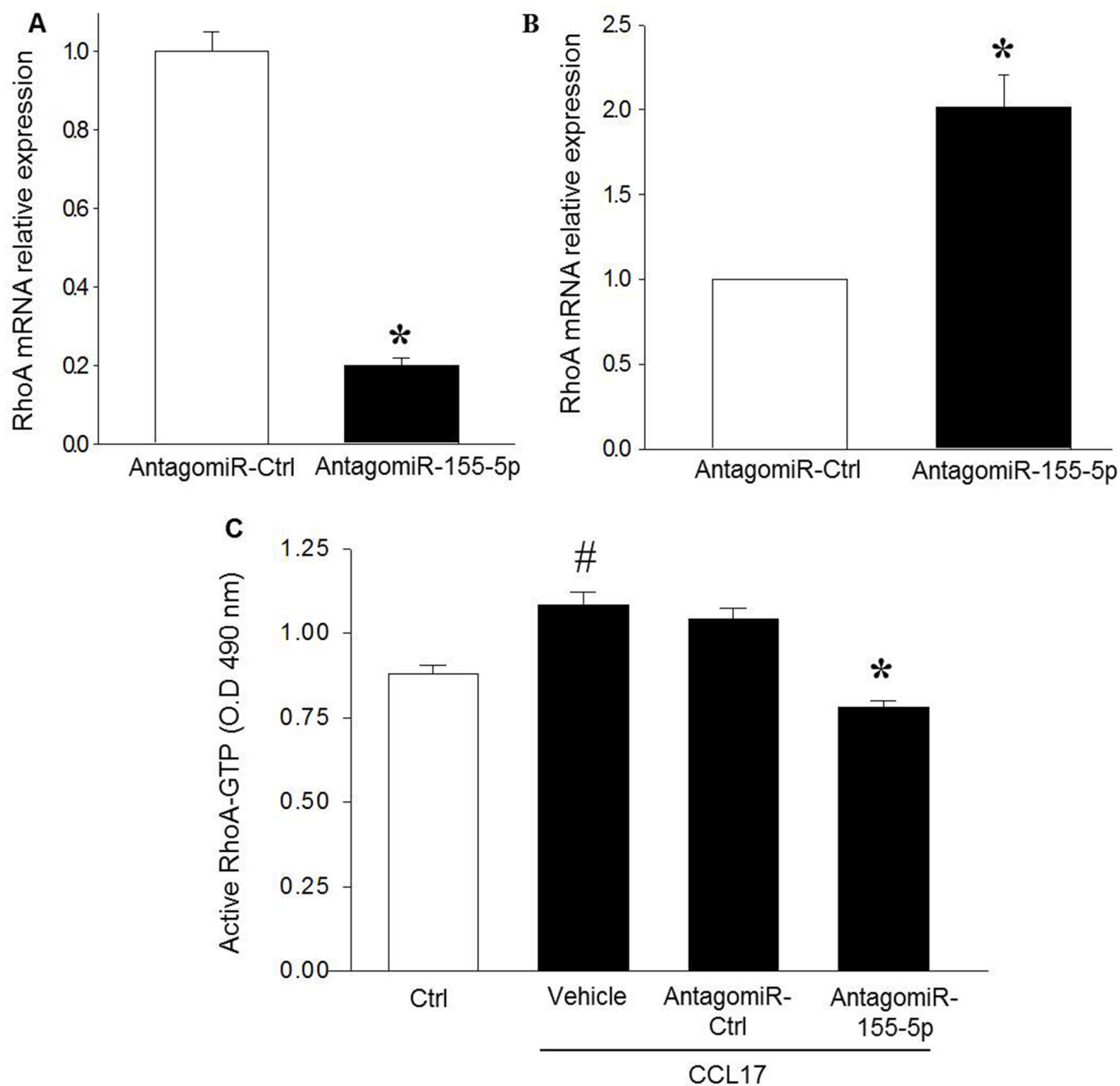

Figure 2: AntagomiR-155-5p reduces RhoA expression and activity in colon cancer cells. (A) transfection by AntagomiR155-5p (200 nM) downregulates RhoA mRNA expression in serum starved HT-29 colon cancer cells. Relative expression was demonstrated using qRT-PCR where beta actin was used as housekeeping gene and expression determined using $2^{-\Delta \Delta C T}$ method. (B) QRT-PCR of RhoA mRNA expression in response to miR-155-5p knockdown in serum-grown HT-29 cells. Relative expression was normalized to beta actin and determined using $2^{-\triangle \triangle C T}$ method. Data represents mean $\pm \mathrm{SEM}$ and $(n=4)$. ${ }^{*} P<0.05$ versus AntagomiR control. (C) AntagomiR-155-5p $(200 \mathrm{nM})$ reduced CCL17-induced RhoA activation in serum starved HT-29 colon cancer cells. Data represent mean \pm SEM and $n=4$. ${ }^{\#} P<0.05$ versus negative ctrl and $* P<0.05$ versus AntagomiR ctrl. 
be present in the cytoplasm as miRNA-ribonucleoprotein complex (miRNPs) and their functions are mediated by Ago2 protein [25], RIP assays were performed on AntagomiR-155-5p transfected HT-29 cells to determine the association between miR-155-5p and RhoA mRNA. Levels of mRNA levels in the immunoprecipitates were detected by use of qRT-PCR. We observed that miR- 155-5p was preferentially enriched (109-folds) in Ago2containing miRNPs relative to ctrl-IgG immunoprecipitates (Figure 4A). In contrast, enrichment of miR-155-5p was reduced by $81 \%$ in AntagomiR-155-5p transfected HT-29 carcinoma cells (Figure 4A). Moreover, the anti-Ago2 antibody specifically pulled down nearly 5-fold more RhoA mRNA as compared to ctrl-IgG. Knocking down miR-155-
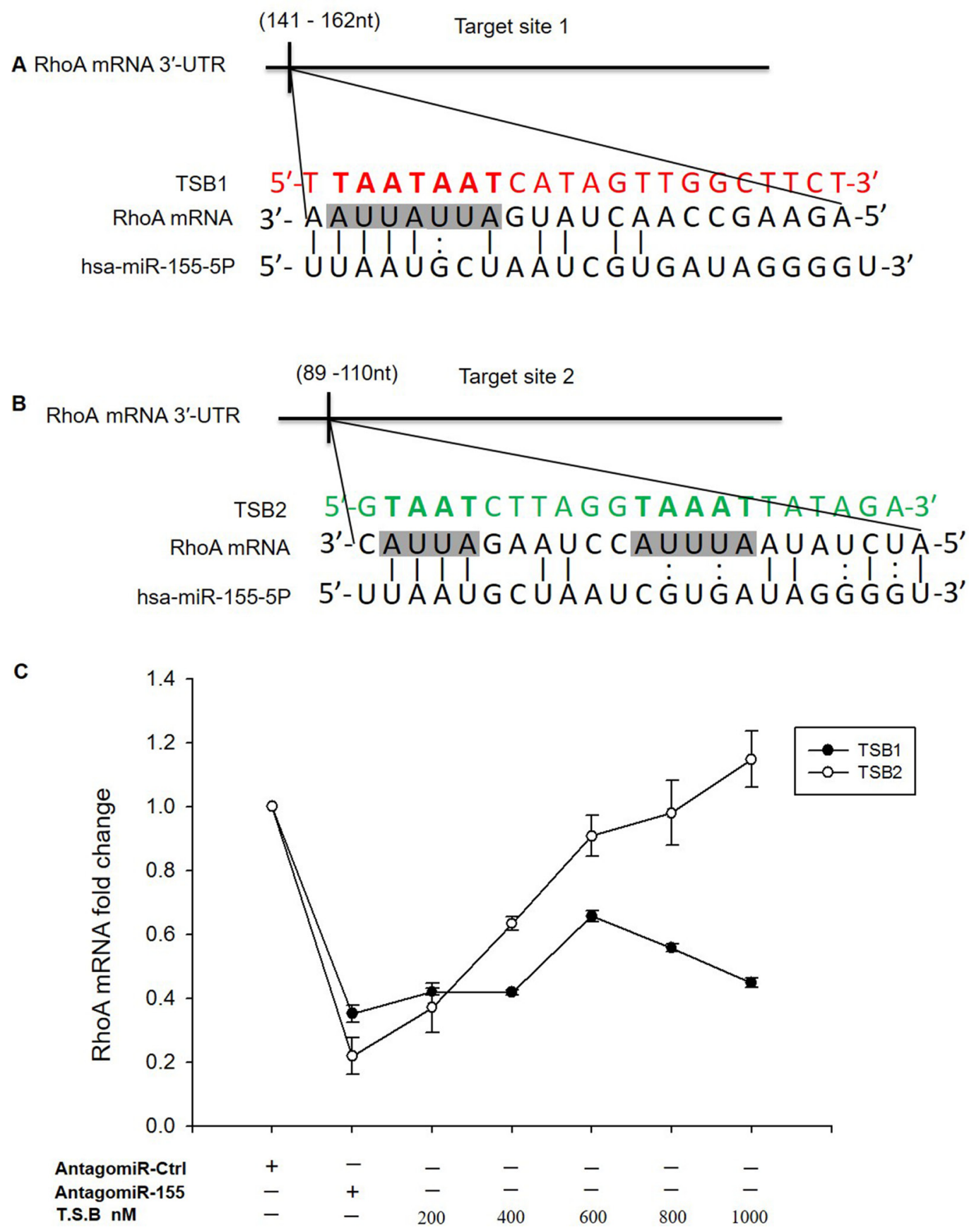

Figure 3: RhoA is a direct target of miR-155-5p. (A) predicted target site 1 of miR-155-5p in RhoA mRNA 3'-UTR sequence containing an ARE (AUUA) motif in shaded box. The seeding region of miR-155-5p complementary to AUUA was blocked using TSB1, red sequence. (B) target site 2 of miR-155-5p in the RhoA mRNA 3'-UTR sequence is depicted in shaded boxes containing two AREs motifs, AUUA and AUUUA, blocked by TSB2, green sequence. (C) TSB2 dose-dependently reversed the effect of AntagomiR-155-5p on RhoA mRNA expression in serum starved HT-29 colon cancer cells. Data represent mean \pm SEM and $n=3$. 
$5 p$ reduced Ago2 antibody-induced enrichment of RhoA mRNA by $58 \%$ compared to AntagomiR Ctrl (Figure 4B).

\section{DISCUSSION}

Tumor cell migration is a precondition for subsequent metastasis to distant organs [26]. In the present study, we examined posttranscriptional regulation of chemokine-induced colon cancer cell migration. We found that inhibition of miR-155-5p decreased RhoA activity and migration of HT-29 carcinoma cells in response to CCL17 stimulation. Moreover, we identified a functional target site of miR-155-5p at the 3'-UTR of RhoA mRNA in HT29 cells. To our knowledge, this is the first report showing that miRNAs can directly regulate chemokine-induced colon cancer cell migration and suggest that targeting miR-155 might be a useful strategy to ameliorate the spread of colon cancer cells to distant sites.

MiRNAs have emerged as an important class of short noncoding RNAs regulating gene expression as well as an array of cellular functions, such as differentiation, growth, proliferation, adhesion, migration and apoptosis $[27,28]$. MiR-155 has been reported to be overexpressed in colon cancer and previous studies have shown that RhoA is a direct target of miR-155 in different cell types, including breast cancer, endothelial, and epithelial cells [17, 29-34]. Considering the key role of RhoA in cancer cell migration $[6,35]$, it was of great interest to examine the role of miR-155 in CCL17-induced colon cancer cell migration in the present study. We found that AntagomiRmediated knock down of miR-155-5p decreased CCL17dependent cell migration without affecting colon cancer cell viability, suggesting that miR-155-5p is an important

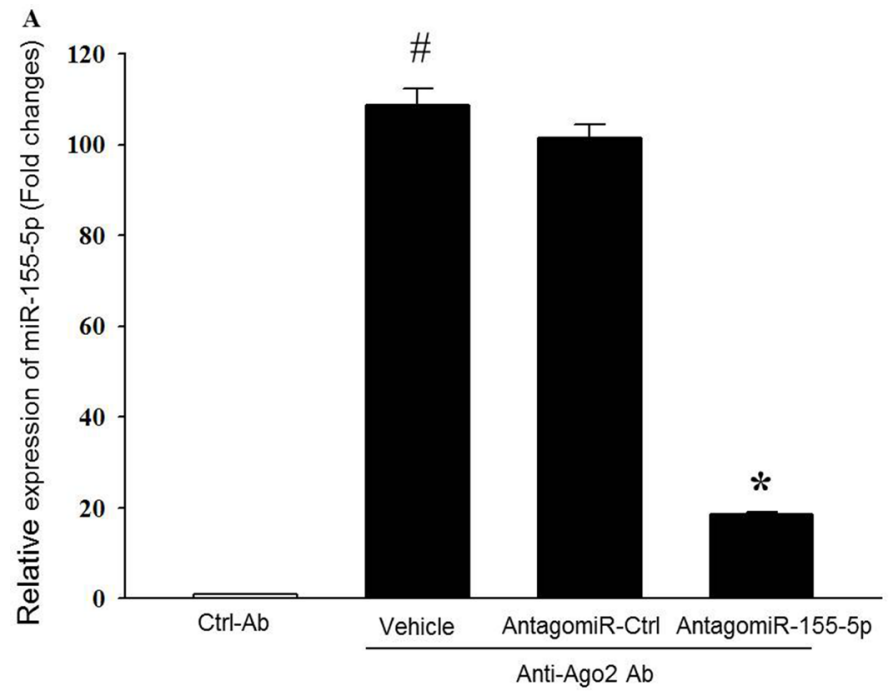

regulator of chemokine-induced directed colon cancer cell chemotaxis. This finding extends on a previous study showing that overexpression of miR-155 in HT-29 cells causes increased random migration [31]. Indeed, this adds colon cancer cells to the line of tumor cells, including hepatocellular, squamous, renal, pancreatic, and breast cancer cells, which exhibits miR-155-dependent migration [19, 36-38]. In this context, it is interesting to note that increased expression of miR-155 is associated with higher frequency of distant metastases in patients with colorectal cancer [31]. Thus, it could be speculated that miR-155-dependent tumor cell migration might at least be one mechanism explaining the increased risk of metastasis in patients with high expression of miR155. Moreover, we observed that AntagomiR-induced knock down of miR-155-5p also attenuated RhoA mRNA expression and activity, suggesting that miR-155-5p is a positive regulator of RhoA in serum starved colon cancer cells. In general, miRNAs are considered to inhibit gene translation; however, accumulating data demonstrate that several miRNAs can cause increased RNA translation in cells under quiescence-like conditions, such as high cell confluence or serum starvation [13, 15]. Besides cellular conditions, different cell types respond differently to specific miRNAs. For example, miR-21 has been demonstrated to upregulate $\mathrm{Bcl}-2$ in pancreatic carcinoma cells but downregulates Bcl-2 expression in breast and glioblastoma cancer cells [39]. Such context-dependent translation of some miRNAs targets is supported by our findings showing that knocking down miR-155-5p in non-serum starved colon cancer cells triggered increased expression of RhoA mRNA (Figure 2B and Supplementary Figure 1C). Considered together, these findings suggest

B

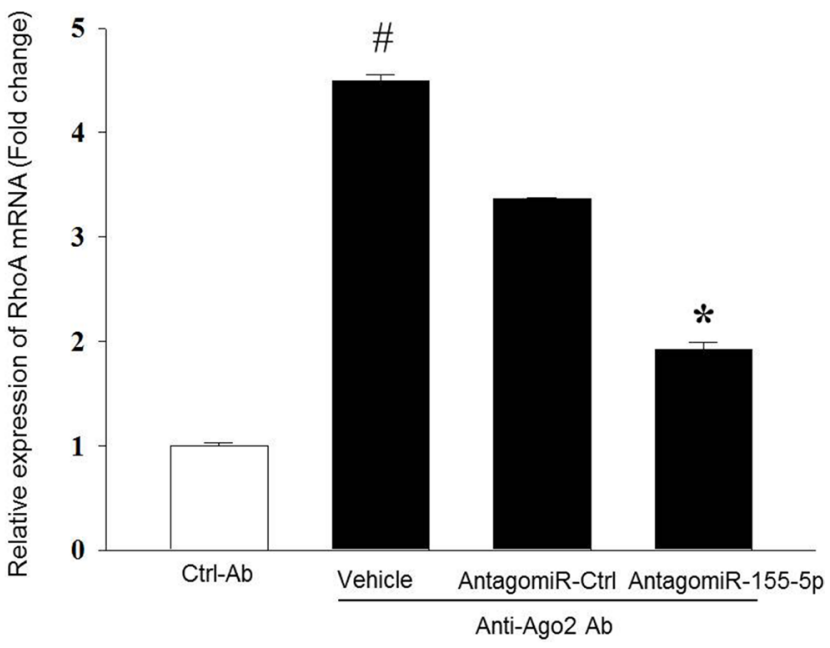

Figure 4: MiR-155-5p is associated with RhoA mRNA in Ago2 immunoprecipitates. The amount of miR-155-5p and RhoA mRNA was measured in input RNA used for the RIP assays by qRT-PCR. AntagomiR-155-5p decreased relative enrichment of (A) miR155-5p and (B) RhoA mRNA in Ago2 immunoprecipitates. Data are expressed as mean \pm SEM and $n=4$. Data are expressed as fold change compared to anti-IgG ctrl. U1 snRNA and beta actin were used as housekeeping genes for miR-155-5p and RhoA mRNA, respectively. ${ }^{\#} P<0.05$ versus ctrl-Ab and $* P<0.05$ versus anti-Ago2-AntagomiR ctrl treated cells. 
that miR-155-5p constitutes a pro-carcinogenic miRNA in serum starved colon cancer cells via promotion of RhoA signaling and migration in response to CCL17 stimulation. To our knowledge, this study is the first report to show that $\mathrm{miR}-155-5 \mathrm{p}$ regulates colon cancer cell migration in response to chemokine signaling.

It is widely held that RhoA acts as a pro-oncogene and is often overexpressed in different kinds of tumors, including colon cancer [40]. RhoA plays an important function as a molecular switch in transducing extracellular signals to actin and microtubule cytoskeleton as an integrated part of cell migration [41]. As described above, we found that miR-155-5p is a positive regulator of RhoA mRNA expression and function in serum starved colon cancer cells. Positive regulation of mRNA translation by miRNAs is related to $3^{\prime}$-UTR target sites of miRNAs during cell cycle arrest [13-15]. In order to determine whether RhoA is a direct target of miR-155$5 \mathrm{p}$, we performed bioinformatics analysis and found five target sites using RNAhybrid in the 3'-UTR of RhoA mRNA, showing that RhoA mRNA has multiple sites for miR-155 as described before [30, 32, 33]. However, our work was focused on ARE motifs specifically (AUUA and AUUUA) in the RhoA mRNA sequence considering that published data have shown that AREs present in the $3^{\prime}$-UTR of mRNAs could play a central role in activating RNA translation [12, 13, 18, 42]. We identified one region that was complementary to the seeding region of miR155-5p with $4^{\prime}$-mer perfect binding (TS1) and another region that had both perfect at the seeding region and imperfect binding at its centered site (TS2). Previous reports indicated that binding to imperfect centered sites is as important as binding to seeding sites in augmenting miRNA and mRNA target interactions through imperfect binding [43]. We then designed specific blockers targeting these ARE sites at 3'-UTR of RhoA mRNA. Interestingly, we found co-incubation with one specific blocker targeting the TS2 ARE motifs (AUUA and AUUUA), which binds miR-155-5p at the seeding region and the centered site, dose-dependently reversed AntagomiR-155-5p-induced inhibition of RhoA mRNA expression, suggesting that this specific ARE region of 3'-UTR of RhoA mRNA is a functional target of miR-155-5p in serum starved colon cancer cells. Thus, this study identifies a novel target site regulating translational activation of RhoA mRNA by miR-155-5p. These results are in line with our findings showing that serum starved HT-29 colon cancer cells exhibited enrichment of RhoA mRNA and miR-155$5 p$ levels in the Ago 2 protein. It is important to note that miRNAs might act on multiple gene targets. In this context, it has been shown that GW182, which is a key protein in miRNA-mediated translation repression and part of the RISC complex, promotes miRNA induced translation in G0 state [44], and whether miR-155-5p could negatively regulate GW182 and thereby initiate translation activation remains to be elucidated. Increased translation can also be mediated by AREs-binding proteins, such as human antigen $\mathrm{R}$ (Hur), cytotoxic granule-associated RNA binding protein, and Tristetraprolin via direct or indirect miRNA binding [45-47]. For example, it has been reported that miR-155 increase tumor necrosis factor alpha mRNA stability and transcription via Hur in activated macrophages [48]. Knowing that the Hur protein is overexpressed in colon cancer cells it might be of value to study the role of Hur in mediating miR-155-induced translation of RhoA mRNA in serum starved colon cancer cells in future studies to improve the understanding of mechanisms regulating colon cancer cell migration and metastasis.

In summary, our data show that miR-155-5p positively regulates CCL17-induced RhoA activity and migration of serum starved colon cancer cells. Moreover, this effect of miR-155-5p was found to be mediated by specific ARE elements, AUUA and AUUUA, present in the $3^{\prime}$-UTR region of RhoA mRNA. These data do not only elucidate novel mechanisms regulating chemokinedependent migration of colon cancer cells but might also help to develop more specific and effective strategies against colon cancer cell metastasis.

\section{MATERIALS AND METHODS}

\section{Cells and reagents}

The human epithelial colon adenocarcinoma cell line HT-29 was obtained from American Type Culture Collection (HTB-38, ATCC, Manassas, VA, USA). Cells were cultured in Dulbecco's Modified Eagle Medium (DMEM); (Sigma-Aldrich, Stockholm, Sweden), supplemented with $10 \%$ FBS, $100 \mathrm{U} / \mathrm{ml}$ penicillin, $100 \mu \mathrm{g} / \mathrm{ml}$ streptomycin at $37^{\circ} \mathrm{C}$ and $5 \% \mathrm{CO}_{2}$. AntagomiR ctrl and AntagomiR-155-5p inhibitor (Life Technologies, Carlsbad, CA, USA) were used to evaluate the role of miR-155-5p by use of lipofectamine RNAimax transfection reagent (Life Technologies, Carlsbad, CA, USA). Chemokine CCL17 was purchased from Peprotech (Rocky Hill, NJ, USA). Anti-Ago2 clone 1B1-E2H5 RIP-certified and ctrl-IgG were purchased from (MBL international, Woburn, MA, USA). Target site blockers (TSB) LNA oligonucleotides were from Exiqon $\mathrm{A} / \mathrm{S}$ (Vedbaek, Denmark).

\section{Cell transfection}

HT-29 colon cancer cells at $60 \%$ confluency were starved overnight and on the next day $1 \times 10^{6}$ cells were plated in a 6 -well culture plate. Cells were reverse transfected with AntagomiR-155-5p (100 nM and 200 $\mathrm{nM}$ ) or AntagomiR ctrl for $24 \mathrm{~h}$ using Lipofectamine RNAiMax (Life Technologies, Carlsbad, CA, USA) in OptiMEM reduced serum media according to manufacturer's instructions. After $24 \mathrm{~h}$, cells were harvested and expression 
of miR-155-5p and RhoA mRNA was analyzed by qRTPCR. Briefly, RNA samples were extracted using Directzol RNA extraction kit (Zymo Research, Irvine, CA, USA) kit according to manufacturer's recommendations. $100 \mathrm{ng}$ total RNA was used in each reaction and cDNA was synthesized using Mir- $\mathrm{X}^{\mathrm{TM}}$ miRNA First-Strand Synthesis Kit and miR-155-5p and RhoA mRNA were quantified using mir-X ${ }^{\mathrm{TM}}$ miRNA qRT-PCR SYBR ${ }^{\circledR}$ kit (Clontech, Mountain View, CA, USA). The PCR primers used were as follows; hsa-miR-155-5p specific sense 5'GGGTTAATGCTAATCGTGATAGGGGT-3', RhoA sense; 5'-AGAGGTGTATGTGCCCACAGTGTT-3', antisense; 5'-AGGCGATCATAATCTTCCTGCCCA-3', U6 snRNA sense; GCTTCGGCAGCACATATACTA, antisense; CGA ATTTGCGTGTCATCCTTG, Beta actin sense; 5'-AGAG CCTCGCCTTTGCCGATCC-3', antisense; 5'-CACATG CCGGAGCCGTTGTCG-3'. Expression of RhoA relative to beta actin, and miR-155-5p relative to U6 snRNA were determined using $2^{-\Delta \Delta} \mathrm{CT}$ method.

\section{Trypan blue dye exclusion assay}

Viability of HT-29 colon cancer cells was quantitatively measured using trypan blue exclusion assay. After transfection by AntagomiR-155-5p (200 nM) and AntagomiR Ctrl at $24 \mathrm{~h}$, cell suspensions were mixed with $0.4 \%$ Trypan blue stain. Total cells and viable cells (cells that excluded the blue stain) were counted using hemocytometer under a light microscope. All assays were performed in quadruplicate.

\section{Chemotaxis assay}

Chemotactic response of HT-29 cells was evaluated by using 24-well cell migration chambers with $8 \mu \mathrm{m}$ pore size inserts (Corning Coster, Corning, NY, USA). The Colon cancer cells were serum starved overnight and resuspended in Opti-MEM serum reduced media. The Cells were then transfected with either AntagomiR-155-5p (200 nM) or AntagomiR ctrl $(200 \mathrm{nM})$ for 24 h. $1 \times 10^{6}$ cells $/ \mathrm{ml}$ were loaded in the inserts. DMEM with or without $100 \mathrm{ng} / \mathrm{ml}$ of CCL17 was added to the lower chambers and incubated for $3 \mathrm{~h}$ at $37^{\circ} \mathrm{C}\left(5 \% \mathrm{CO}_{2}\right)$. Non-migrated cells were removed by cotton swabs from the upper surface of the insert and cells on the lower surface of the insert membrane were fixed in ice-cold $100 \%$ methanol and stained with $0.5 \%$ crystal violet. All migrated cells were counted microscopically in at least 5 different fields. Migration index was then calculated as the ratio of the number of migrated cells divided by the number of cells in the control wells.

\section{RhoA activation assay}

RhoA-GTP activity was measured using the G-LISA RhoA activation assay Biochem kit (Cytoskeleton Inc., Denver, CO, USA) according to manufacturer's instructions. Briefly, cells were serum starved overnight and reverse transfected by either AntagomiR-155-5p $(200 \mathrm{nM})$ or AntagomiR-155 ctrl for $24 \mathrm{~h}$. The next day, cells were stimulated with $100 \mathrm{ng} / \mathrm{ml}$ of CCL17 for $3 \mathrm{~h}$ and cells were trypsinized, washed by ice cold PBS and lysed in $0.35 \mathrm{ml}$ lysis buffer of the kit on ice for $10 \mathrm{~min}$. Then, cells were homogenized using a 20 -gauge needle for 20 strokes on ice and centrifuged at $10000 \mathrm{rpm}$ for $20 \mathrm{~min}$ at $4^{\circ} \mathrm{C}$. Supernatants were collected, snap frozen in liquid nitrogen and stored at $-80^{\circ} \mathrm{C}$ until used. $50 \mu \mathrm{L}$ of the supernatants were used for protein determination using Precision Red Advanced Protein Assay supplied with the kit (Cytoskeleton Inc., Denver, CO, USA). $1 \mathrm{mg}$ / $\mathrm{ml}$ of protein was used for quantitative detection of active RhoA according to the manufacturer's recommendations. Absorbance was read at $490 \mathrm{~nm}$ using a microplate ELISA reader.

\section{Target site prediction and target site blockers (TSB) of miR-155-5p}

RNAhybrid web-based bioinformatics target prediction algorithm was used to predict binding sites for miR-155-5p at the 3'-UTR of RhoA mRNA (http:// bibiserv.techfak.uni-bielefeld.de/rnahybrid). However, we took into consideration the presence of regulatory ARE sites in RhoA mRNA specifically AUUUA and AUUA motifs and therefore our analysis was limited to ARE sites. To assess the function of the binding sites, we designed target site blockers, TSB1 and TSB2, (22 nucleotides) to bind selectively to sequences overlapping with the miR155-5p ARE sites in the 3'-UTR of RhoA mRNA. The blockers were synthesized as fully phosphorothiolated Locked Nucleic acids in the DNA sequences to increase their affinity and selectivity for the target. Under serum starved conditions, the target site blockers TSB1_RhoA miR-155-5p; 5'-TTAATAATCATAGTTGGCTTCT-3' and TSB2_RhoA_miR-155-5p; 5'-GTAATCTTAGGTAAATT ATAGA-3' were co-transfected with the AntagomiR-155$5 \mathrm{p}$ in different concentrations $(200-1000 \mathrm{nM})$ in HT-29 colon cancer cells. RhoA mRNA levels were quantified using qRT-PCR. The potential target was functionally validated using RNA immunoprecipitation (RIP) assays.

\section{RIP assay}

RIP has recently been utilized to identify mRNAs that are associated with the RNA-silencing machinery and therefore being targets of cellular miRNAs [23]. RIP was performed using the EZ-Magna RIP kit (Millipore, Billerica, MA, USA) following the manufacturer's protocol. Cells were scraped off $24 \mathrm{~h}$ after transfection with either AntagomiR-155-5p (200 nM) or AntagomiR ctrl. Cells were then lysed in complete RIP lysis buffer containing protease inhibitor cocktail, after which 100 $\mu l$ of whole cell extract was incubated with RIP buffer containing magnetic beads conjugated with an anti- 
Ago2 antibody or ctrl-IgG antibody and rotated for $3 \mathrm{~h}$ at $4^{\circ} \mathrm{C}$. After several washes samples were incubated with Proteinase $\mathrm{K}$ with shaking to digest proteins at $55^{\circ} \mathrm{C}$. RNA was then isolated and concentrations were measured using a NanoDrop (ND-1000, Spectrophotometer, Thermo Scientific, Waltham, MA, USA). The co-immunoprecipitated (co-IP) RNA, including microRNA:mRNA complexes, were analysed by qRT-PCR to measure relative enrichment of miR-155-5p and RhoA mRNA.

\section{Statistical analysis}

All statistical analyses were performed using SigmaPlot ${ }^{\circledR} 10$ software. For multiple comparisons Kruskal-Wallis One Way Analysis of variance on ranks followed by the Dunnett's post hoc test was used. $P$-value $<0.05$ was considered significant.

\section{ACKNOWLEDGMENTS AND FUNDING}

This study was supported by the Swedish Medical Research Council (2012-3685), Einar och Inga Nilssons stiftelse, Greta och Johan Kocks stiftelser, Magnus Bergvalls stiftelse, Mossfelts stiftelse, Malmö University Hospital Cancer Foundation, Malmö University Hospital and Lund University.

\section{CONFLICTS OF INTEREST}

The authors declare that they have no conflicts of interest.

\section{REFERENCES}

1. Ferlay J, Soerjomataram I, Dikshit R, Eser S, Mathers C, Rebelo M, Parkin DM, Forman D, Bray F. Cancer incidence and mortality worldwide: sources, methods and major patterns in GLOBOCAN 2012. International journal of cancer. 2015; 136:E359-86. doi: 10.1002/ijc.29210.

2. Usher-Smith JA, Walter FM, Emery JD, Win AK, Griffin SJ. Risk Prediction Models for Colorectal Cancer: A Systematic Review. Cancer prevention research. 2016; 9:13-26. doi: 10.1158/1940-6207.

3. Vatandoust S, Price TJ, Karapetis CS. Colorectal cancer: Metastases to a single organ. World J Gastroenterol. 2015; 21:11767-76. doi: 10.3748/wjg.v21.i41.11767.

4. Vicari AP, Caux C. Chemokines in cancer. Cytokine Growth Factor Rev. 2002; 13:143-54.

5. Singh R, Lillard JW, Jr., Singh S. Chemokines: key players in cancer progression and metastasis. Frontiers in bioscience. 2011; 3: 1569-82. http://dx.doi.org/10.2741/246.

6. Al-haidari AA, Syk I, Jirstrom K, Thorlacius H. CCR4 mediates CCL17 (TARC)-induced migration of human colon cancer cells via RhoA/Rho-kinase signaling. Int $\mathrm{J}$ Colorectal Dis. 2013; 28:1479-87. doi: 10.1007/s00384013-1712-y.
7. Olkhanud PB, Baatar D, Bodogai M, Hakim F, Gress R, Anderson RL, Deng J, Xu M, Briest S, Biragyn A. Breast cancer lung metastasis requires expression of chemokine receptor CCR4 and regulatory T cells. Cancer Research. 2009; 69:5996-6004. doi: 10.1158/0008-5472.CAN-08-4619.

8. Ridley AJ. Rho GTPase signalling in cell migration. Current opinion in cell biology. 2015; 36:103-12. doi: 10.1016/j. ceb.2015.08.005.

9. Ender C, Meister G. Argonaute proteins at a glance. Journal of Cell Science. 2010; 123:1819-23. doi: 10.1242/ jes.055210.

10. Gregory RI, Chendrimada TP, Cooch N, Shiekhattar R. Human RISC couples microRNA biogenesis and posttranscriptional gene silencing. Cell. 2005; 123:631-40. doi: 10.1016/j. cell.2005.10.022.

11. Larne O, Östling P, Haflidadóttir BS, Hagman Z, Aakula A, Kohonen P, Kallioniemi O, Edsjö A, Bjartell A, Lilja H, Lundwall $\AA$, Ceder Y. miR-183 in prostate cancer cells positively regulates synthesis and serum levels of prostatespecific antigen. Eur Urol. 2015; 68:581-8. doi: 10.1016/j. eururo.2014.12.025.

12. Vasudevan S. Posttranscriptional upregulation by microRNAs. Wiley Interdiscip Rev RNA. 2012; 3:311-30. doi: 10.1002/wrna.121.

13. Vasudevan $\mathrm{S}$, Tong Y, Steitz JA. Switching from repression to activation: microRNAs can up-regulate translation. Science. 2007; 318:1931-4. doi: 10.1126/science.1149460.

14. Truesdell SS, Mortensen RD, Seo M, Schroeder JC, Lee JH, LeTonqueze O, Vasudevan S. MicroRNA-mediated mRNA translation activation in quiescent cells and oocytes involves recruitment of a nuclear microRNP. Sci Rep. 2012; 2:842. doi: 10.1038/srep00842.

15. Vasudevan S, Tong Y, Steitz JA. Cell-cycle control of microRNA-mediated translation regulation. Cell Cycle. 2008; 7:1545-9. doi: 10.4161/cc.7.11.6018.

16. Faraoni I, Antonetti FR, Cardone J, Bonmassar E. miR155 gene: a typical multifunctional microRNA. Biochim Biophys Acta. 2009; 1792:497-505. doi: 10.1016/j. bbadis.2009.02.013.

17. Zhang GJ, Xiao HX, Tian HP, Liu ZL, Xia SS, Zhou T. Upregulation of microRNA-155 promotes the migration and invasion of colorectal cancer cells through the regulation of claudin-1 expression. Int J Mol Med. 2013; 31:1375-80. doi: 10.3892/ijmm.2013.1348.

18. Vasudevan S, Steitz JA. AU-rich-element-mediated upregulation of translation by FXR1 and Argonaute 2. Cell. 2007; 128:1105-18. doi: 10.1016/j.cell.2007.01.038.

19. Huang $\mathrm{C}$, Li H, Wu W, Jiang T, Qiu Z. Regulation of miR-155 affects pancreatic cancer cell invasiveness and migration by modulating the STAT3 signaling pathway through SOCS1. Oncology Reports. 2013; 30:1223-30. doi: 10.3892/or.2013.2576.

20. Jiang S, Zhang HW, Lu MH, He XH, Li Y, Gu H, Liu MF, Wang ED. MicroRNA-155 functions as an OncomiR in breast cancer by targeting the suppressor of cytokine 
signaling 1 gene. Cancer Research. 2010; 70:3119-27. doi: 10.1158/0008-5472.CAN-09-4250.

21. Jiang YX, Du ZM, Jiao L, Shao Q, Fu S, Shao JY, Zhu XF, Ernberg I5, Li YH. Inhibition of MiR-155 suppresses cell migration in nasopharyngeal carcinoma through targeting ZDHHC2. Int J Clin Exp Med. 2015; 8:8472-84.

22. Liu F, Kong X, Lv L, Gao J. MiR-155 targets TP53INP1 to regulate liver cancer stem cell acquisition and selfrenewal. FEBS letters. 2015; 589:500-6. doi: 10.1016/j. febslet.2015.01.009.

23. Keene JD, Komisarow JM, Friedersdorf MB. RIP-Chip: the isolation and identification of mRNAs, microRNAs and protein components of ribonucleoprotein complexes from cell extracts. Nat Protoc. 2006; 1:302-7. doi: 10.1038/nprot.2006.47.

24. Murphy D, Dancis B, Brown JR. The evolution of core proteins involved in microRNA biogenesis. BMC Evol Biol. 2008; 8:92. doi: 10.1186/1471-2148-8-92.

25. Valinezhad Orang A, Safaralizadeh R, KazemzadehBavili M. Mechanisms of miRNA-Mediated Gene Regulation from Common Downregulation to mRNASpecific Upregulation. Int J. Genomics. 2014; $2014: 970607$. doi: $10.1155 / 2014 / 970607$.

26. Gassmann P, Enns A, Haier J. Role of tumor cell adhesion and migration in organ-specific metastasis formation. Onkologie. 2004; 27:577-82. doi: 10.1159/000081343.

27. Jansson MD, Lund AH. MicroRNA and cancer. Mol Oncol. 2012; 6:590-610. doi: 10.1016/j.molonc.2012.09.006.

28. Rothschild SI. microRNA therapies in cancer. Mol Cell Ther. 2014; 2:7. doi: 10.1186/2052-8426-2-7.

29. Bijkerk R, de Bruin RG, van Solingen C, Duijs JM, Kobayashi K, van der Veer EP, ten Dijke P, Rabelink TJ, Goumans MJ, van Zonneveld AJ. MicroRNA-155 functions as a negative regulator of RhoA signaling in TGF-betainduced endothelial to mesenchymal transition. Microrna. 2012; 1:2-10.

30. Kong W, Yang H, He L, Zhao JJ, Coppola D, Dalton WS, Cheng JQ. MicroRNA-155 is regulated by the transforming growth factor beta/Smad pathway and contributes to epithelial cell plasticity by targeting RhoA. Mol Cell Biol. 2008; 28:6773-84. doi: 10.1128/MCB.00941-08.

31. Qu YL, Wang HF, Sun ZQ, Tang Y, Han XN, Yu XB, Liu K. Up-regulated miR-155-5p promotes cell proliferation, invasion and metastasis in colorectal carcinoma. Int J Clin Exp Pathol. 2015; 8:6988-94.

32. Tang J, Ahmad A, Sarkar FH. The role of microRNAs in breast cancer migration, invasion and metastasis. Int J Mol Sci. 2012; 13:13414-37. doi: 10.3390/ijms131013414.

33. Tian R, Wang RL, Xie H, Jin W, Yu KL. Overexpressed miRNA-155 dysregulates intestinal epithelial apical junctional complex in severe acute pancreatitis. World $\mathrm{J}$ Gastroenterol. 2013; 19:8282-91. doi: 10.3748/wjg.v19. i45.8282.

34. Weber M, Kim S, Patterson N, Rooney K, Searles CD. MiRNA-155 targets myosin light chain kinase and modulates actin cytoskeleton organization in endothelial cells. Am J Physiol Heart Circ Physiol. 2014; 306:H1192203. doi: 10.1152/ajpheart.00521.2013.

35. O'Connor K, Chen M. Dynamic functions of RhoA in tumor cell migration and invasion. Small GTPases. 2013; 4:141-7. doi: $10.4161 /$ sgtp. 25131 .

36. Han ZB, Chen HY, Fan JW, Wu JY, Tang HM, Peng ZH. Upregulation of microRNA-155 promotes cancer cell invasion and predicts poor survival of hepatocellular carcinoma following liver transplantation. J Cancer Res Clin Oncol. 2012; 138:153-61. doi: 10.1007/s00432-011-1076-z.

37. Johansson J, Berg T, Kurzejamska E, Pang MF, Tabor V, Jansson M, Roswall P, Pietras K, Sund M, Religa P, Fuxe J. MiR-155-mediated loss of C/EBPbeta shifts the TGF-beta response from growth inhibition to epithelial-mesenchymal transition, invasion and metastasis in breast cancer. Oncogene. 2013; 32:5614-24. doi: 10.1038/onc.2013.322.

38. Li S, Chen T, Zhong Z, Wang Y, Li Y, Zhao X. microRNA-155 silencing inhibits proliferation and migration and induces apoptosis by upregulating BACH1 in renal cancer cells. Mol Med Rep. 2012; 5:949-54. doi: 10.3892/mmr.2012.779.

39. Dong J, Zhao YP, Zhou L, Zhang TP, Chen G. Bcl-2 upregulation induced by miR-21 via a direct interaction is associated with apoptosis and chemoresistance in MIA PaCa-2 pancreatic cancer cells. Arch Med Res. 2011; 42: 8-14. doi: 10.1016/j.arcmed.2011.01.006.

40. Wang H, Zhao G, Liu X, Sui A, Yang K, Yao R, Wang Z, Shi Q. Silencing of RhoA and RhoC expression by RNA interference suppresses human colorectal carcinoma growth in vivo. J Exp Clin Cancer Res. 2010; 29:123. doi: 10.1186/1756-9966-29-123.

41. Sit ST, Manser E. Rho GTPases and their role in organizing the actin cytoskeleton. Journal of Cell Science. 2011; 124:679-83. doi: 10.1242/jcs.064964.

42. Khabar KS. Post-transcriptional control during chronic inflammation and cancer: a focus on AU-rich elements. Cell Mol Life Sci. 2010; 67:2937-55. doi: 10.1007/s00018-010-0383-x.

43. Martin HC, Wani S, Steptoe AL, Krishnan K, Nones K, Nourbakhsh E, Vlassov A, Grimmond SM, Cloonan N. Imperfect centered miRNA binding sites are common and can mediate repression of target mRNAs. Genome biology. 2014; 15:R51. doi: 10.1186/gb-2014-15-3-r51.

44. Yang Z, Jakymiw A, Wood MR, Eystathioy T, Rubin RL, Fritzler MJ, Chan EK. GW182 is critical for the stability of GW bodies expressed during the cell cycle and cell proliferation. Journal of Cell Science. 2004; 117:5567-78. doi: 10.1242/jcs.01477.

45. Kawai T, Lal A, Yang X, Galban S, Mazan-Mamczarz K, Gorospe M. Translational control of cytochrome c by RNAbinding proteins TIA-1 and HuR. Mol Cell Biol. 2006; 26:3295-307. doi: 10.1128/MCB.26.8.3295-3307.2006.

46. Poria DK, Guha A, Nandi I, Ray PS. RNA-binding protein HuR sequesters microRNA-21 to prevent translation 
repression of proinflammatory tumor suppressor gene programmed cell death 4. Oncogene. 2016; 35:1703-15. doi: 10.1038/onc.2015.235.

47. Wang J, Guo Y, Chu H, Guan Y, Bi J, Wang B. Multiple functions of the RNA-binding protein $\mathrm{HuR}$ in cancer progression, treatment responses and prognosis. Int J Mol Sci. 2013; 14:10015-41. doi: 10.3390/ijms140510015.
48. Bala S, Marcos M, Kodys K, Csak T, Catalano D, Mandrekar P, Szabo G. Up-regulation of microRNA-155 in macrophages contributes to increased tumor necrosis factor \{alpha\} (TNF $\{$ alpha\}) production via increased mRNA half-life in alcoholic liver disease. J Biol Chem. 2011; 286:1436-44. doi: 10.1074/jbc.M110.145870. 